\title{
Positive Solutions for System of Nonlinear Fractional Differential Equations in Two Dimensions with Delay
}

\author{
Azizollah Babakhani \\ Department of Basic Science, Babol University of Technology, Babol 47148-71167, Iran \\ Correspondence should be addressed to Azizollah Babakhani, babakhani@nit.ac.ir \\ Received 2 November 2009; Revised 20 January 2010; Accepted 31 March 2010 \\ Academic Editor: Dumitru Baleanu \\ Copyright (C) 2010 Azizollah Babakhani. This is an open access article distributed under the \\ Creative Commons Attribution License, which permits unrestricted use, distribution, and \\ reproduction in any medium, provided the original work is properly cited. \\ We investigate the existence and uniqueness of positive solution for system of nonlinear fractional \\ differential equations in two dimensions with delay. Our analysis relies on a nonlinear alternative \\ of Leray-Schauder type and Krasnoselskii's fixed point theorem in a cone.
}

\section{Introduction}

Fractional differential equations have gained considerable importance due to their varied applications [1-5] in viscoelasticity, electroanalytical chemistry, and many physical problems [1]. So far there have been several fundamental works on the fractional derivative and fractional differential equations, written by Oldham and Spanier [3], Miller and Ross [2], Podlubny [1], and others. These works are an introduction to the theory of the fractional derivative and fractional differential equations and provide a systematic understanding of the fractional calculus such as the existence and the uniqueness, some analytic methods for solving fractional differential equations, namely, Green's function method, the Mellin transform method, and the power series.

Control systems subject to delays have been extensively studied [6] and the delay differential equations are large and important class of dynamic systems. They often arise in either natural or technological control problems. Equations with discontinuity often appear in various control theory models $[7,8]$. Time delay, always existing in real systems, usually results in oscillations around the discontinuity surface. Shustin [9] has studied various aspects of oscillations for the system of differential equations with the delay

$$
\dot{x}(t)=F_{i}\left(x_{1}(t), \ldots, x_{n}(t), t\right)-\operatorname{sign} x_{i}\left(t-h_{i}\right), \quad i=1, \ldots, n,
$$


in dynamics of oscillations in a multidimension, where $F_{1}, \ldots, F_{n} \in C^{1}\left(\mathbb{R}^{n+1}\right)$ and $h_{1}, \ldots, h_{n}$ are positive constants.

Roy et al. developed a control methodology for linear time-invariant plants that uses multiple delayed observations in feedback [10]. Fractional differential systems have proved to be useful in control processing for the last two decades [11, 12].

As explained in the above text regarding the ordinary differential equations in control theory and others, we can modify the applications of ordinary differential equations to ordinary fractional differential equations.

The existence and uniqueness of solution for the system of fractional differential equation have been studied in the papers $[13,14]$. As a pursuit in this paper, we discuss the existence and uniqueness of positive solutions for system of nonlinear fractional differential equations in two-dimensional with the delay

$$
\begin{array}{cc}
D^{\alpha}[x(t)-x(0)]=f\left(t, x_{t}, y_{t}\right), & t \in(0, T], \\
D^{\beta}[y(t)-y(0)]=g\left(t, x_{t}, y_{t}\right), & t \in(0, T], \\
(x(t), y(t))=(\phi(t), \psi(t)), & t \in[-\tau, 0],
\end{array}
$$

where

(i) $0<\alpha, \beta<1$ and $D^{\alpha}, D^{\beta}$ are the Riemann-Liouville fractional derivatives,

(ii) $f, g:(0, T] \times C^{2} \rightarrow[0,+\infty)$ are given continuous functions so that $\lim _{t \rightarrow 0^{+}} f\left(t, x_{t}, y_{t}\right)=\lim _{t \rightarrow 0^{+}} g\left(t, x_{t}, y_{t}\right)=+\infty$ (i.e., $f$ and $g$ are singular at $t=0$ ) and $C=C\left([-\tau, 0], \mathbb{R}^{\geq 0}\right)$,

(iii) $x_{t}, y_{t} \in C$, where $x_{t}(s)=x(t+s), y_{t}(s)=y(t+s), s \in[-\tau, 0], t \in(0, T]$.

The paper is organized as follows. In Section 2, we give definitions of the fractional derivative and fractional integral with some basic properties. Required topics of functional analysis were also introduced. Section 3 deals with existence of positive solution theorem and gives an illustrative example. The unique positive solution theorem with an example has been discussed in Section 4.

\section{Basic Definitions and Preliminaries}

We recall some standard definitions and results $[1-4,15,16]$. In the following $\Pi$ and $\Sigma$ are real Banach spaces and $W$ is an operator (not necessarily linear) with domain in $\Pi$ and range in $\Sigma$.

Definition 2.1. A subset $K$ of $\Pi$ is called a cone if the following conditions hold well:

(i) the set $K$ is closed,

(ii) if $f, g \in K, \alpha>0, \beta>0$ implies that $\alpha f+\beta g \in K$,

(iii) if $f \in K,-f \in K$ implies that $f=\theta$ which is the zero element of $\Pi$.

Note:

Every cone $K$ induces a semiordering in $\Pi$, namely, $\mathrm{f} \prec g$ if $g-f \in K$. 
Definition 2.2. For $f, g \in E$ the order interval $\langle f, g\rangle$ is defined as [17]

$$
\langle f, g\rangle=\{h \in E: f \leq h \leq g\}
$$

Definition 2.3. A subset $E \subset \Pi$ is called order bounded if $E$ is contained in some order interval.

Definition 2.4. A cone $K$ is called normal if there exists a positive constant $\mu$ such that $f, g \in V$ and $\theta \prec f \prec g$ implies that $\|f\| \leq \mu\|g\|$.

We state the two fixed point results which will be needed in this paper. Our first result is a nonlinear alternative of Leray-Schauder type in a cone whereas our second is Krasnoselskii`s fixed point theorem.

Theorem 2.5 (Leray-Schauder Theorem). Let $E$ be a Banach space with $C \subseteq E$ closed and convex. Assume that $U$ is relatively open subset of $C$ with $0 \in U$ and $W: \bar{U} \rightarrow C$ is a continuous, compact map. Then either

(i) W has fixed point in $\bar{U}$ or

(ii) there exist $u \in \partial U$ and $\gamma \in(0,1)$ with $u=\gamma W u$.

Theorem 2.6 (Krasnoselskii's fixed point theorem [16]). Let $E=(E,\|\cdot\|)$ be a Banach space and let $K \subset E$ be a cone in $E$. Assume that $\Omega_{1}$ and $\Omega_{2}$ are open subsets of $E$ with $0 \in \Omega_{1}$ and $\bar{\Omega}_{1} \subset \Omega_{2}$ and let $W: K \cap\left(\bar{\Omega}_{2} \backslash \Omega_{1}\right) \rightarrow K$ be continuous and completely continuous. In addition suppose that either

(i) $\|W u\| \leq\|u\|$ for $u \in K \cap \partial \Omega_{1}$ and $\|W u\| \geq\|u\|$ for $u \in K \cap \Omega_{2}$ or

(ii) $\|W u\| \leq\|u\|$ for $u \in K \cap \partial \Omega_{1}$ and $\|W u\| \leq\|u\|$ for $u \in K \cap \Omega_{2}$.

Then, $W$ has a fixed point in $K \cap\left(\bar{\Omega}_{2} \backslash \Omega_{1}\right)$.

In this paper the Beta function $B(\alpha, \beta)$ is used also. $B(\alpha, \beta)$ is closely related to the Gamma function [1]. If $\alpha, \beta>0$, then it has the integral representation

$$
B(\alpha, \beta)=\int_{0}^{1} t^{\alpha-1}(1-t)^{\beta-1} d t
$$

It may be written in terms of the Gamma function as $B(\alpha, \beta)=\Gamma(\alpha) \Gamma(\beta) / \Gamma(\alpha+\beta)$.

Definitions of Riemann-Liouville fractional derivative/integral and their properties are given bellow [1-4].

Definition 2.7. Let $x:[a, b] \rightarrow \mathbb{R}$ and $x \in L^{1}[a, b]$ then the expression

$$
I_{a^{+}}^{\alpha} x(t)=\frac{1}{\Gamma(\alpha)} \int_{a}^{t}(t-s)^{\alpha-1} x(t) d t, \quad x>a
$$

is called a left-sided fractional integral of order $\alpha$. 
Definition 2.8. Let $n$ be a positive integer number and $\alpha \in(n-1, n]$. The left-sided fractional derivative of a function $x:[a, b] \rightarrow \mathbb{R}$ is defined as

$$
D_{a^{+}}^{\alpha} x(t)=D^{n}\left(I^{n-\alpha} x(t)\right), \quad t \in[a, b]
$$

We denote $D_{a^{+}}^{\alpha} x(t)$ as $D_{a}^{\alpha} x(t)$ and $I_{a^{+}}^{\alpha} x(t)$ as $I_{a}^{\alpha} x(t)$. Further $D_{0^{+}}^{\alpha} x(t)$ and $I_{0^{+}}^{\alpha} x(t)$ are referred to as $D^{\alpha} x(t)$ and $I^{\alpha} x(t)$, respectively.

If the fractional derivative $D_{a}^{\beta} x(t)$ is integrable then

$$
\begin{aligned}
I_{a}^{\alpha}\left(D_{a}^{\beta} x(t)\right)= & I_{a}^{\alpha-\beta} x(t)-\left\{I_{a}^{n-\beta} x(t)\right\}_{t=a} \frac{(t-a)^{\alpha-n}}{\Gamma(\alpha-n+1)} \\
& -\sum_{j=1}^{n-1}\left\{D_{a}^{\beta-j} x(t)\right\}_{t=a} \frac{(t-a)^{\alpha-j}}{\Gamma(\alpha-j+1)}
\end{aligned}
$$

where $n-1 \leq \beta \leq \alpha<n$ and $n \in \mathbb{N}[1]$. Further, if $x \in C[a, b]$, then $\left\{I_{a}^{n-\beta} x(t)\right\}_{t=a}=0$ and then (2.5) implies that

$$
I_{a}^{\alpha}\left(D_{a}^{\alpha} x(t)\right)=x(t), \quad 0<\alpha<1
$$

\section{Existence Theorem}

In this section we discuss the system of nonlinear fractional differential equation (1.2) which has at least one positive solution.

Lemma 3.1 (see [18]). Let $h:(0, T] \rightarrow \mathbb{R}$ be a continuous function and $\lim _{t \rightarrow 0^{+}} h(t)=+\infty$. If there exits $\sigma \in(0,1)$ such that $0<\sigma<\alpha$ and $t^{\sigma} h(t)$ is a continuous function on $[0, T]$, then $H(t)=I^{\alpha} t^{\sigma} h(t)$ is continuous $[0, T]$.

In the following theorem we want to prove that (1.2) is equivalent to a system of integral equations.

Theorem 3.2. Suppose that $f, g:(0, T] \times C^{2} \rightarrow[0,+\infty)$ are given continuous functions with $\lim _{t \rightarrow 0} f\left(t, x_{t}, y_{t}\right)=+\infty=\lim _{t \rightarrow 0} g\left(t, x_{t}, y_{t}\right)=+\infty$. If there exist $\rho, \sigma \in(0,1)$ such that $0<\rho, \sigma<\alpha$ and $t^{\rho} f\left(t, x_{t}, y_{t}\right), t^{\sigma} g\left(t, x_{t}, y_{t}\right)$ are continuous functions on $[0, T]$, then (1.2) is equivalent to the system of integral equations

$$
\begin{gathered}
x(t)=x(0)+I^{\alpha} f\left(t, x_{t}, y_{t}\right), \quad t \in(0, T], \\
y(t)=y(0)+I^{\beta} g\left(t, x_{t}, y_{t}\right), \quad t \in(0, T], \\
x(t)=\phi(t) \geq 0, \quad y(t)=\psi(t) \geq 0, \quad t \in[-\tau, 0] .
\end{gathered}
$$


Proof. It is sufficient to prove that the first equation of system equation (1.2) is equivalent to the first equation of system equation (3.1). So,

$$
I^{\alpha}\left\{D^{\alpha}[x(t)-x(0)]\right\}=I^{\alpha} f\left(t, x_{t}, y_{t}\right), \quad t \in(0, T] .
$$

Using (2.6) we get $I^{\alpha}\left\{D^{\alpha}[x(t)-x(0)\}=x(t)-x(0)\right.$. Hence (3.2) implies that $x(t)=x(0)+$ $I^{\alpha} f\left(t, x_{t}, y_{t}\right), t \in(0, T]$.

Conversely, first we note that $I^{\alpha} f(t, x(t), y(t))=I^{\alpha}\left\{t^{\rho} t^{-\rho} f\left(t, x_{t}, y_{t}\right)\right\}$ exists by Lemma 3.1. $D^{\alpha} I^{\alpha} f\left(t, x_{t}, y_{t}\right)=f\left(t, x_{t}, y_{t}\right)$ as $t^{-\rho} f\left(t, x_{t}, y_{t}\right)$ is continuous and $I^{\alpha} f\left(t, x_{t}, y_{t}\right) \in$ $C[0, T]$. Therefore, the system of fractional integral equation (3.1) is a solution of (1.2).

Let $\omega:[-\tau, T] \rightarrow[0,+\infty) \times[0,+\infty)$ be a function defined by

$$
\omega(t)= \begin{cases}(\phi(0), \psi(0)), & t \in[0, T], \\ (\phi(t), \psi(t)), & t \in[-\tau, 0],\end{cases}
$$

for each $z(t)=(\eta(t), \gamma(t))$ with $z(0)=(0,0)$ where $\eta$ and $\gamma$ belong to $C([0, T], \mathbb{R})$. We introduce the notation $\bar{z}$ defined by

$$
\bar{z}(t)= \begin{cases}(\eta(t), \gamma(t)), & t \in[0, T], \\ (0,0), & t \in[-\tau, 0] .\end{cases}
$$

We can decompose $(x(\cdot), y(\cdot))$ as $(x(t), y(t))=\bar{z}(t)+\omega(t), t \in[-\tau, T]$ which implies that $\left(x_{t}, y_{t}\right)=\bar{z}_{t}+\omega_{t}, t \in[0, T]$. Hence, by Theorem 3.2, (1.2) is equivalent to the system of integral equations

$$
\begin{aligned}
& \eta(t)=I^{\alpha} f\left(t, \bar{z}_{t}+\omega_{t}\right), \\
& \gamma(t)=I^{\beta} g\left(t, \bar{z}_{t}+\omega_{t}\right),
\end{aligned}
$$

where $t \in[0, T]$. Set $\Omega=\{z=(\eta(t), \gamma(t)): \eta, \gamma \in C([0, T], \mathbb{R}), \eta(0)=\gamma(0))=0\}$. For each $z \in \Omega$, let $\|z\|_{T}$ be the seminorm in $\Omega$ defined by

$$
\|z\|_{T}=\|z(0)\|+\|z\|=\|z\|=\sup \{|\eta(t)|,|\gamma(t)|: t \in[0, T]\} .
$$

$\Omega$ is a Banach space with norm $\|\cdot\|_{T}$. Let $K$ be a cone of $\Omega$,

$$
\begin{gathered}
K=\{z=(\eta(t), \gamma(t)) \in \Omega: \eta(t) \geq 0, \gamma(t) \geq 0, t \in[0, T]\}, \\
K^{*}=\left\{\left((x, y): x, y \in C\left([-\tau, T], \mathbb{R}^{+}\right)\right),(x(t), y(t))=(\phi(t) \geq 0, \psi(t) \geq 0), t \in[-\tau, 0]\right\} .
\end{gathered}
$$

For each $t \in[0, T]$, we define the operator $W: K \rightarrow K$ by

$$
W z(t)=\left(I^{\alpha} f\left(t, \bar{z}_{t}+\omega_{t}\right), I^{\beta} g\left(t, \bar{z}_{t}+\omega_{t}\right)\right)=(F \eta(t), G \gamma(t))
$$


where

$$
F \eta(t)=I^{\alpha} f\left(t, \bar{z}_{t}+\omega_{t}\right), \quad G \eta(t)=I^{\beta} g\left(t, \bar{z}_{t}+\omega_{t}\right)
$$

Lemma 3.3. Let $f\left(t, x_{t}, y_{t}\right), g\left(t, x_{t}, y_{t}\right)$, and $t \in(0, T]$ be nonnegative continuous functions, where $x_{t}, y_{t} \in C$. Then, the operator $W$ with the following conditions is maps-bounded set into bounded sets in $K$.

(H1) $0<\lim _{t \rightarrow 0^{+}} f\left(t, x_{t}, y_{t}\right)=\lim _{t \rightarrow 0^{+}} g\left(t, x_{t}, y_{t}\right)=+\infty$

(H2) if there exist $\rho, \sigma \in(0,1)$ such that $\rho, \sigma<\alpha, t^{\rho} f\left(t, x_{t}, y_{t}\right)$, and $t^{\sigma} g\left(t, x_{t}, y_{t}\right)$ are continuous on $[0, T]$.

Proof. By assumptions of Lemma 3.3 and by using Lemma 3.1, it is clear that $W z(t)=$ $(F \eta(t), \gamma(t)) \in K$. There exist positive constants $\Lambda_{1}, \Lambda_{2}$ such that $\left|t^{\rho} f\left(t, x_{t}, y_{t}\right)\right| \leq \Lambda_{1}$ and $\left|t^{\sigma} g\left(t, x_{t}, y_{t}\right)\right| \leq \Lambda_{2}$, as $t^{\rho} f\left(t, x_{t}, y_{t}\right)$ and $t^{\sigma} g\left(t, x_{t}, y_{t}\right)$ are continuous on $[0, T] \times C^{2}$. Hence,

$$
\begin{aligned}
I^{\alpha} f\left(t, x_{t}, y_{t}\right) & =I^{\alpha}\left(t^{-\rho} t^{\rho} f\left(t, x_{t}, y_{t}\right)\right) \\
& \leq \Lambda_{1} I^{\alpha} t^{-\rho}=\frac{\Lambda_{1}}{\Gamma(\alpha)} \int_{0}^{t}(t-s)^{\alpha-1} s^{-\rho} d s \\
& =\frac{\Lambda_{1} t^{\alpha-\rho}}{\Gamma(\alpha)} B(1-\rho, \alpha) \leq \frac{\Lambda_{1} T^{\alpha-\rho}}{\Gamma(\alpha)} B(1-\rho, \alpha) .
\end{aligned}
$$

Similarly,

$$
I^{\beta} g\left(t, x_{t}, y_{t}\right) \leq \frac{\Lambda_{2} T^{\beta-\sigma}}{\Gamma(\beta)} B(1-\sigma, \beta)
$$

Let $H \subset K$ be bounded, that is, for each $z=(\eta, \gamma) \in H$ there exist positive constants $L$ such that $\|z\|_{T} \leq L$. In view of (3.10), (3.11) we have

$$
\begin{aligned}
& \left|F_{\eta}(t)\right| \leq\left|I^{\alpha} f\left(t, \bar{z}_{t}+\omega_{t}\right)\right| \leq \frac{\Lambda_{1} T^{\alpha-\rho}}{\Gamma(\alpha)} B(1-\rho, \alpha), \\
& \left|G_{\gamma}(t)\right| \leq\left|I^{\alpha} f\left(t, \bar{z}_{t}+\omega_{t}\right)\right| \leq \frac{\Lambda_{2} T^{\beta-\sigma}}{\Gamma(\alpha)} B(1-\sigma, \beta) .
\end{aligned}
$$

Therefore, $\|W z(t)\| \leq \Lambda$ where

$$
\Lambda=\max \left\{\frac{\Lambda_{1} T^{\alpha-\rho}}{\Gamma(\alpha)} B(1-\rho, \alpha), \frac{\Lambda_{2} T^{\beta-\sigma}}{\Gamma(\beta)} B(1-\sigma, \beta)\right\}
$$

Hence, $W(K)$ is bounded. 
Lemma 3.4. Suppose that $f\left(t, x_{t}, y_{t}\right), g\left(t, x_{t}, y_{t}\right), x_{t}, y_{t} \in C, t \in(0, T]$ are continuous functions and further two conditions (H1) and (H2) are satisfied. Then, the operator $W$ is continuous and completely continuous.

Proof. In the following we want to prove that $W$ is continuous. It is sufficient to prove that $F$ and $G$ are continuous. Let $z_{0}=\left(u_{0}, v_{0}\right) \in K$ with $\left\|u_{0}\right\|=c_{0}$ and $\left\|v_{0}\right\|=d_{0}$. If $z=(u, v) \in K$ with $\left\|u-u_{0}\right\|<l_{1}$ and $\left\|v-v_{0}\right\|<l_{2}$, then $\|u\|<l_{1}+c_{0}$ and $\|v\|<l_{2}+d_{0}$. By the continuity of $t^{\rho} f\left(t, \bar{z}_{t}+\omega_{t}\right)$ and $t^{\sigma} g\left(t, \bar{z}_{t}+\omega_{t}\right)$ on $[0, T]$, we conclude that $t^{\rho} f\left(t, \bar{z}_{t}+\omega_{t}\right)$ and $t^{\sigma} g\left(t, \bar{z}_{t}+\omega_{t}\right)$ are uniformly continuous on $[0, T] \times\left[0, c_{0}\right] \times\left[0, d_{0}\right]$. Therefore, $\left|F u(t)-F u_{0}(t)\right|,\left|G v(t)-G v_{0}(t)\right|, t \in$ $[0, T]$ are continuous and hence,

$$
\left\|W z(t)-W z_{0}(t)\right\|=\max \left\{\left|F u(t)-F u_{0}(t)\right|,\left|G v(t)-G v_{0}(t)\right|\right\} .
$$

is continuous.

Finally, we want to prove that the operator $W$ is equicontinuous. Let $H \subset K$ be bounded. Suppose that, $z=(u, v) \in K$ and $t, r \in[0, T]$ such that $t<r$. For given $\epsilon>0$, there exists $\delta_{1}>0$, so that if $|t-r|<\delta_{1}$, then $\left|t^{\rho} f\left(t, \bar{z}_{t}+\omega_{t}\right)-t^{\rho} f\left(t, \bar{z}_{r}+\omega_{r}\right)\right|<\epsilon$ :

$$
\begin{aligned}
\mid F u(t) & -F u(r)|=| I^{\alpha}\left\{f\left(t, \bar{z}_{t}+\omega_{t}\right)-f\left(r, \bar{z}_{r}+\omega_{r}\right)\right\} \mid \\
\leq & \frac{1}{\Gamma(\alpha)}\left|\int_{0}^{t}(t-s)^{\alpha-1} s^{-\rho} s^{\rho} f\left(s, \bar{z}_{s}+\omega_{s}\right) d s-\int_{0}^{r}(r-s)^{\alpha-1} s^{-\rho} s^{\rho} f\left(s, \bar{z}_{s}+\omega_{s}\right) d s\right| \\
\leq & \frac{1}{\Gamma(\alpha)}\left|\int_{0}^{r}(t-s)^{\alpha-1} s^{-\rho} s^{\rho} f\left(s, \bar{z}_{s}+\omega_{s}\right)-\int_{0}^{r}(r-s)^{\alpha-1} s^{-\rho} s^{\rho} f\left(s, \bar{z}_{s}+\omega_{s}\right) d s\right| \\
& +\frac{1}{\Gamma(\alpha)}\left|\int_{t}^{r}(t-s)^{\alpha-1} s^{-\rho} s^{\rho} f\left(s, \bar{z}_{s}+\omega_{s}\right) d s\right| \\
\leq & \frac{1}{\Gamma(\alpha)} \int_{0}^{r}\left|(t-s)^{\alpha-1} s^{-\rho}-(r-s)^{\alpha-1} s^{-\rho}\right| \times\left|s^{\rho} f\left(s, \bar{z}_{s}+\omega_{s}\right)-s^{\rho} f\left(s, \bar{z}_{s}+\omega_{s}\right)\right| d s \\
& +\frac{1}{\Gamma(\alpha)} \int_{t}^{r}\left|(t-s)^{\alpha-1} s^{-\rho}\right|\left|s^{\rho} f\left(s, \bar{z}_{s}+\omega_{s}\right)\right| d s \\
< & \frac{\epsilon}{\Gamma(\alpha)} \int_{0}^{r}\left\{(r-s)^{\alpha-1} s^{-\rho}+(r-s)^{\alpha-1} s^{-\rho}\right\} d s+\frac{\Lambda_{1}}{\Gamma(\alpha)} \int_{t}^{r}(s-t)^{\alpha-1} s^{-\rho} d s \\
= & \frac{2 \epsilon}{\Gamma(\alpha)} B(1-\rho, \alpha)+\frac{\Lambda_{1}}{\Gamma(\alpha)} \int_{t}^{r}(s-t)^{\alpha-1} s^{-\rho} d s .
\end{aligned}
$$

Similarly, for given $\epsilon>0$, there exists $\delta_{2}>0$, so that if $|t-r|<\delta_{2}$, then

$$
|G u(t)-G u(r)| \leq \frac{2 \epsilon}{\Gamma(\beta)} B(1-\sigma, \beta)+\frac{\Lambda_{2}}{\Gamma(\beta)} \int_{t}^{r}(s-t)^{\beta-1} s^{-\sigma} d s
$$


Case 1. If $t=0$ and $0<r<\delta_{i}, i=1,2$, then

$$
\int_{t}^{r}(s-t)^{\alpha-1} s^{-\rho} d s \leq \max _{0 \leq r \leq T} \int_{0}^{r} s^{\alpha-\rho-1} d s \leq \frac{T^{\alpha-\rho}}{\alpha-\rho} .
$$

Set

$$
\begin{aligned}
& \lambda_{1}=\max \left\{\frac{1}{2 \Gamma(\alpha)} B(1-\rho, \alpha), \frac{2 \Lambda_{1}}{\epsilon(\alpha-\rho)} T^{\alpha-\rho}\right\} \\
& \lambda_{2}=\max \left\{\frac{1}{2 \Gamma(\beta)} B(1-\sigma, \beta), \frac{2 \Lambda_{2}}{\epsilon(\beta-\sigma)} T^{\beta-\sigma}\right\} .
\end{aligned}
$$

Hence, $|F u(t)-F u(r)|<\lambda_{1} \epsilon$ and $|G v(t)-G v(r)|<\lambda_{2} \epsilon$.

Case 2. If $0<t<r<\delta_{i} \leq T, i=1,2$, then

$$
m_{1}=\frac{\Lambda_{1}}{\Gamma(\alpha)} \int_{t}^{r}(s-t)^{\alpha-1} s^{-\rho} d s, \quad m_{2}=\frac{\Lambda_{2}}{\Gamma(\beta)} \int_{t}^{r}(s-t)^{\beta-1} s^{-\sigma} d s
$$

are finite. Set

$$
\mu_{1}=\max \left\{\frac{1}{2 \Gamma(\alpha)} B(1-\rho, \alpha)\right\}, \quad \mu_{2}=\max \left\{\frac{1}{2 \Gamma(\beta)} B(1-\sigma, \beta)\right\} .
$$

Hence, $|F u(t)-F u(r)|<\mu_{1} \epsilon$ and $|G v(t)-G v(r)|<\mu_{2} \epsilon$. Thus, for any given $\epsilon>0$, for all $z=(u, v) \in K$ and for all $t, r \in[0, T]$ with $|r-t|<\delta$ where $\delta=\min \left\{\delta_{1}, \delta_{2}\right\}$, for the Euclidian distance $d$ on $\mathbb{R}^{2}$ we have

$$
d(W z(t), W z(r))=\left\{[F u(r)-F u(t)]^{2}\right\}^{1 / 2}+\left\{[G v(r)-G v(t)]^{2}\right\}^{1 / 2}<\eta \sqrt{2} \epsilon
$$

where $\eta=\max \left\{\mu_{1}, \mu_{2}, \lambda_{1}, \lambda_{2}\right\}$. Therefore, $W(H)$ is equicontinuous and the Ascoli-Arzela theorem implies that $\overline{W(H)}$ is compact and hence $W: K \rightarrow K$ is completely continuous.

Theorem 3.5. If $f\left(t, x_{t}, y_{t}\right)$ and $g\left(t, x_{t}, y_{t}\right)$ on $(0, T] \times C^{2}$ are nonnegative continuous functions, then the system of nonlinear fractional differential equation (1.2) with the conditions (H1) and (H2) has at least one positive solution $x^{*} \in K^{*}$, satisfying $\left\|x^{*}\right\| \leq \max \{\|\phi\|,\|\psi\|, h\}$, where $h$ is observed in the proof of the theorem.

Proof. By Lemma 3.3, the operator $W: K \rightarrow K$ is continuous and completely continuous. We prove that there exits an open set $U \subseteq K$, with $z \neq \gamma W(z)$ for $\gamma \in(0,1)$ and $z \in \partial U$. Let $z=(u, v) \in K$ be any solution of $z=\gamma W(z), \gamma \in(0,1)$. In view of Theorem 3.5, we have

$$
z(t)=\gamma W z(t)=\gamma(F u(t), G v(t)), \quad t \in[0, T]
$$


Hence,

$$
z(t)=\gamma W z(t)=\left(\gamma I^{\alpha} t^{-\rho} t^{\rho} f\left(t, \bar{z}_{t}+\omega_{t}\right), \gamma I^{\beta} t^{-\sigma} t^{\sigma} g\left(t, \bar{z}_{t}+\omega_{t}\right)\right)
$$

There exist positive constants $N_{1}, N_{2}$ such that $\left|t^{\rho} f\left(t, \bar{z}_{t}+\omega_{t}\right)\right| \leq N_{1}$ and $\left|t^{\rho} g\left(t, \bar{z}_{t}+\omega_{t}\right)\right| \leq N_{2}$, as $t^{\rho} f\left(t, \bar{z}_{t}+\omega_{t}\right)$ and $t^{\sigma} g\left(t, \bar{z}_{t}+\omega_{t}\right)$ are continuous on $[0, T] \times(C[-\tau, T])^{2}$. Hence,

$$
\begin{aligned}
& |u(t)| \leq \frac{N_{1}}{\Gamma(\alpha)} \max _{0 \leq t \leq T} t^{\alpha-\rho} B(1-\rho, \alpha) \leq \frac{N_{1} T^{\alpha-\rho}}{\Gamma(\alpha)} B(1-\rho, \alpha), \\
& |v(t)| \leq \frac{N_{2}}{\Gamma(\beta)} \max _{0 \leq t \leq T} t^{\beta-\sigma} B(1-\sigma, \beta) \leq \frac{N_{2} T^{\beta-\sigma}}{\Gamma(\beta)} B(1-\sigma, \beta) .
\end{aligned}
$$

If we consider

$$
h=1+\max \left\{\frac{N_{1} T^{\alpha-\rho}}{\Gamma(\alpha)} B(1-\alpha, \alpha), \frac{N_{2} T^{\beta-\sigma}}{\Gamma(\beta)} B(1-\beta, \beta)\right\}
$$

then any solution $z=\gamma W(z)$ satisfies $\|z\| \neq h$. Set $U=\{z \in K:\|z\|<h\}$. Theorem 2.5 guarantees that $W$ has fixed point $z \in \bar{U}$. Theorem 3.2 gives that (1.2) has a positive solution $x^{*} \in K^{*}$ satisfying $\left\|x^{*}\right\| \leq \max \{\|\phi\|,\|\psi\|, h\}$.

Example 3.6. Consider the system of nonlinear fractional differential equation

$$
\begin{gathered}
D^{4 / 5}[x(t)-x(0)]=\frac{e^{-t}}{\sqrt{t}}\left(x_{t}+y_{t}\right), \quad t \in(0,1], \\
D^{3 / 4}[y(t)-y(0)]=\frac{e^{-t}}{\sqrt[4]{t}}\left(x_{t}+y_{t}\right), \quad t \in(0,1], \\
(x(t), y(t))=\left(-\sin \pi t,-\sin \left(\frac{\pi t}{2}\right)\right), \quad t \in[-1,0],
\end{gathered}
$$

where

$$
\begin{aligned}
& x_{t}=\int_{-1}^{-t} \frac{d s}{1+x^{2}(t+s)}=\int_{t-1}^{0} \frac{d s}{1+x^{2}(s)}, \quad t \in[-1,0] \\
& y_{t}=\int_{-1}^{-t} \frac{y^{2}(t+s)}{1+y^{2}(t+s)} d s=\int_{t-1}^{0} \frac{y^{2}(s)}{1+y^{2}(s)} d s, \quad t \in[-1,0] .
\end{aligned}
$$

Here, $\alpha=4 / 5, \beta=3 / 4, f\left(t, x_{t}, y_{t}\right)=e^{-t} t^{-1 / 2}\left(x_{t}+y_{t}\right)$, and $g\left(t, x_{t}, y_{t}\right)=e^{-t} t^{-1 / 4}\left(x_{t}+y_{t}\right)$ with $\lim _{t \rightarrow 0^{+}} f(t, \cdot, \cdot)=+\infty=\lim _{t \rightarrow 0^{+}} g(t, \cdot, \cdot)=+\infty$. If we select $\rho=3 / 4$ and $\sigma=1 / 2$, then $t^{\rho} f(t, \cdot, \cdot)$ and $t^{\sigma} g(t, \cdot, \cdot)$ are continuous on $[0,1]$. 
By Theorem 3.2, the system of nonlinear fractional differential equation (3.26) is equivalent to the system of integral equation

$$
\begin{aligned}
& x(t)=x(0)+I^{4 / 5}\left(e^{-t} t^{-1 / 2}\left(x_{t}+y_{t}\right)\right), \quad t \in(0,1], \\
& y(t)=y(0)+I^{3 / 4}\left(e^{-t} t^{-1 / 4}\left(x_{t}+y_{t}\right)\right), \quad t \in(0,1], \\
& (x(t), y(t))=\left(-\sin \pi t,-\sin \frac{\pi}{2} t\right), \quad t \in[-1,0] .
\end{aligned}
$$

Therefore, by Theorem 3.5, (3.26) has at least one positive solution $x^{*} \in K^{*}$ satisfying $\left\|x^{*}\right\| \leq$ $\max \{\|\phi\|, \psi, h\}$ where

$$
\begin{gathered}
\|\phi\|=\max \left\{\max _{-1 \leq t \leq 0}|-\sin \pi t|, \max _{-1 \leq t \leq 0}\left|-\sin \frac{\pi}{2} t\right|\right\}=1, \\
h=1+\max \left\{\frac{N_{1}}{\Gamma(4 / 5)} B\left(\frac{1}{4}, \frac{4}{5}\right), \frac{N_{2}}{\Gamma(3 / 4)} B\left(\frac{1}{2}, \frac{3}{4}\right)\right\}, \\
N_{1}=\max _{0 \leq t \leq 1}\left\{t^{\rho}\left(e^{-t} t^{-1 / 2}\left(x_{t}+y_{t}\right)\right)\right\}=\max _{0 \leq t \leq 1}\left\{e^{-t} t^{1 / 4}\left(x_{t}+y_{t}\right)\right\} \leq \frac{4}{\sqrt[4]{4 e}}, \\
N_{2}=\max _{0 \leq t \leq 1}\left\{t^{\sigma}\left(e^{-t} t^{-1 / 4}\left(x_{t}+y_{t}\right)\right)\right\}=\max _{0 \leq t \leq 1}\left\{e^{-t} t^{1 / 2}\left(x_{t}+y_{t}\right)\right\} \leq \frac{6}{\sqrt{2 e}} .
\end{gathered}
$$

Note that, for each $t \in[0,1]$, we have $0 \leq x_{t}, y_{t} \leq 2$ and

$$
\max _{0 \leq t \leq 1}\left\{t^{1 / 4} e^{-t}\right\}=\frac{1}{\sqrt[4]{4 e}}, \quad \max _{0 \leq t \leq 1}\left\{t^{1 / 2} e^{-t}\right\}=\frac{1}{\sqrt{2 e}}
$$

Theorem 3.7. Suppose that $f, g:(0, T] \times C^{2} \rightarrow[0, \infty)$ are nonnegative continuous with $\lim _{t \rightarrow+\infty} f\left(t, x_{t}, y_{t}\right)=\lim _{t \rightarrow+\infty} g\left(t, x_{t}, y_{t}\right)=+\infty$. If there exist $\rho, \sigma \in(0,1)$ such that $0<\rho<$ $\alpha, 0<\sigma<\beta$ and $t^{\rho} f\left(t, x_{t}, y_{t}\right)$ and $t^{\sigma} g\left(t, x_{t}, y_{t}\right)$ are continuous on $[0, T] \times C^{2}$, then (1.2) has at least one positive solution with the following conditions:

(H3) $t^{\rho} f\left(t, x_{t}, y_{t}\right) \leq \rho_{1} T^{\rho-\alpha}(\Gamma(1-\rho+\alpha) / \Gamma(1-\rho)), t^{\sigma} g\left(t, x_{t}, y_{t}\right) \leq \rho_{2} T^{\sigma-\beta}(\Gamma(1-\sigma+$ $\beta) / \Gamma(1-\sigma))$,

$\sigma))$,

(H4) $t^{\rho} f\left(t, x_{t}, y_{t}\right) \geq \mu_{1} T^{\rho-\alpha}(\Gamma(1-\rho+\alpha) / \Gamma(1-\rho)), t^{\sigma} g\left(t, x_{t}, y_{t}\right) \geq \mu_{2} T^{\sigma-\beta}(\Gamma(1-\sigma+\beta) / \Gamma(1-$ where $\left(t, x_{t}, y_{t}\right) \in[0, T] \times[0, \rho]^{2},\left(t, x_{t}, y_{t}\right) \in[0, T] \times[0, \mu]^{2}, \rho=\max \left\{\rho_{1}, \rho_{2}\right\}, \mu=\min \left\{\mu_{1}, \mu_{2}\right\}$, and $0<\mu<\rho$. 
Proof. For proving this theorem we provide the conditions required in Theorem 2.6. Set

$$
\begin{aligned}
& \Omega_{1}=\{z=(u, v) \in K:\|z\|<\mu\}, \\
& \Omega_{2}=\{z=(u, v) \in K:\|z\|<\rho\} .
\end{aligned}
$$

For each $z=(u, v) \in K \cap \partial \Omega_{2}$ and $t \in[0, T]$ we have $0 \leq u(t) \leq \rho$ and $0 \leq v(t) \leq \rho$. Condition (H3) implies that

$$
\begin{aligned}
F u(t) & =\frac{1}{\Gamma(\alpha)} \int_{0}^{t}(t-s)^{\alpha-1} s^{-\rho} s^{\rho} f\left(s, \bar{z}_{s}+\omega_{s}\right) d s \\
& \leq \rho_{i} T^{\rho_{1}-\alpha} \frac{\Gamma(1-\rho+\alpha)}{\Gamma(1-\rho)} \frac{1}{\Gamma(\alpha)} \int_{0}^{t}(t-s)^{\alpha-1} s^{-\rho} d s \\
& =\rho_{1} T^{\rho-\alpha} t^{\alpha-\rho} \frac{\Gamma(1-\rho+\alpha)}{\Gamma(1-\rho)} \frac{\Gamma(1-\rho)}{\Gamma(1-\rho+\alpha)}=\rho_{1} T^{\rho-\alpha} t^{\alpha-\rho} .
\end{aligned}
$$

Hence $\|F u\|=\max _{0 \leq t \leq T}|F u(t)| \leq \rho$. Similarly $\|G v\|=\max _{0 \leq t \leq T}|G v(t)| \leq \rho$. Thus, $\|W z\| \leq \rho=$ $\|z\|$ for $z=(u, v) \in K \cap \partial \Omega_{2}$. By using condition (H4) and the above-mentioned proof, we have $\|W z\| \geq \mu=\|z\|$ for $z \in K \cap \partial \Omega_{1}$. Therefore, by Theorem 2.6, (ii), and Theorem 3.2, the proof is completed.

Example 3.8. Consider the system of nonlinear fractional differential equation

$$
\begin{gathered}
D^{1 / 4}[x(t)-x(0)]=\frac{1}{\sqrt[5]{t}} z_{t}, \quad t \in(0,1], \\
D^{1 / 2}[y(t)-y(0)]=\frac{1}{\sqrt[4]{t}} x_{t}, \quad t \in(0,1], \\
D^{3 / 4}[z(t)-z(0)]=\frac{1}{\sqrt{t}} y_{t}, \quad t \in(0,1], \\
x(t)=\left(-\sin \pi t,-\sin \left(\frac{\pi t}{4}\right),-\sin \left(\frac{\pi t}{2}\right)\right), \quad t \in[-1,0],
\end{gathered}
$$


where $x_{t}, y_{t}, z_{t}$ are continuous functions from $[-1,0]$ to $[0,+\infty)$ so that

$$
\begin{aligned}
& x_{t}=\frac{\Gamma(5 / 4)}{\Gamma(3 / 4)}\left\{1+\int_{-1}^{-t} \frac{2 x^{2}(t+s)+1}{1+x^{2}(t+s)} d s\right\}, \\
& y_{t}=\frac{\Gamma(5 / 4)}{\Gamma(1 / 4)}\left\{2+\int_{-1}^{-t} \frac{3 y^{2}(t+s)+2}{1+y^{2}(t+s)} d s\right\}, \\
& z_{t}=\frac{\Gamma(21 / 20)}{\Gamma(4 / 5)}\left\{3+\int_{-1}^{-t} \frac{4 z^{2}(t+s)+3}{1+z^{2}(t+s)} d s\right\} .
\end{aligned}
$$

Here $\quad \alpha=1 / 4, \beta=1 / 2, \gamma=3 / 4, f\left(t, x_{t}, y_{t}, z_{t}\right)=t^{-1 / 5} z_{t}, g\left(t, x_{t}, y_{t}, z_{t}\right)=t^{-1 / 4} x_{t}, h\left(t, x_{t}, y_{t}, z_{t}\right)=$ $t^{-1 / 2} y_{t}$ such that $\lim _{t \rightarrow 0^{+}} f(t, \cdot, \cdot)=\lim _{t \rightarrow 0^{+}} g(t, \cdot, \cdot)=\lim _{t \rightarrow 0^{+}} h(t, \cdot, \cdot)=+\infty$. Also $t^{\rho} f(t, \cdot, \cdot)$, $t^{\sigma} g(t, \cdot, \cdot)$, and $t^{v} h(t, \cdot, \cdot)$ are continuous, where $\rho=1 / 5, \sigma=1 / 4$, and $v=1 / 2$. In the following calculations, we review conditions (H3) and (H4) by using (3.34):

$$
\begin{aligned}
& t^{1 / 5} f\left(t, x_{t}, y_{t}, z_{t}\right)=\frac{\Gamma(21 / 20)}{\Gamma(4 / 5)}\left\{3+\int_{-1}^{-t} \frac{2 z^{2}(t+s)+1}{1+z^{2}(t+s)} d s\right\} \\
& =\frac{\Gamma(21 / 20)}{\Gamma(4 / 5)}\left\{\int_{t-1}^{0} \frac{2 z^{2}(s)+1}{1+z(s)^{2}} d s\right\} \leq \frac{\Gamma(21 / 20)}{\Gamma(4 / 5)}\left\{3+4 \int_{t-1}^{0} d s\right\} \\
& \leq 7 \frac{\Gamma(21 / 20)}{\Gamma(4 / 5)}=7 \frac{\Gamma(1-\rho+\alpha)}{\Gamma(1-\rho)}, \\
& t^{1 / 4} g\left(t, x_{t}, y_{t}, z_{t}\right)=\frac{\Gamma(5 / 4)}{\Gamma(3 / 4)} \int_{-1}^{-t}\left\{1+\frac{2 x^{2}(t+s)+1}{1+x^{2}(t+s)} d s\right\} \\
& =\frac{\Gamma(5 / 4)}{\Gamma(3 / 4)} \int_{t-1}^{0}\left\{1+\frac{2 x_{1}^{2}(s)+1}{1+\left[x_{1}(s)\right]^{2}} d s\right\} \leq \frac{\Gamma(5 / 4)}{\Gamma(3 / 4)}\left\{1+2 \int_{t-1}^{0} d s\right\} \\
& \leq 3 \frac{\Gamma(5 / 4)}{\Gamma(3 / 4)}=3 \frac{\Gamma(1-\sigma+\beta)}{\Gamma(1-\sigma)}, \\
& t^{1 / 2} h\left(t, x_{t}, y_{t}, z_{t}\right)=\frac{\Gamma(5 / 4)}{\Gamma(1 / 4)}\left\{2+\int_{-1}^{-t} \frac{3 y^{2}(t+s)+1}{1+y^{2}(t+s)} d s\right\} \\
& =\frac{\Gamma(5 / 4)}{\Gamma(1 / 4)}\left\{2+\int_{t-1}^{0} \frac{3 x_{2}^{2}(s)+1}{1+\left[x_{2}(s)\right]^{2}} d s\right\} \leq \frac{\Gamma(5 / 4)}{\Gamma(1 / 4)}\left\{2+3 \int_{t-1}^{0} d s\right\} \\
& \leq 5 \frac{\Gamma(5 / 4)}{\Gamma(1 / 4)}=5 \frac{\Gamma(1-v+\gamma)}{\Gamma(1-v)} \text {. }
\end{aligned}
$$


Hence, $\rho=\max \{3,5,7\}=7$ and the above calculations satisfy condition (H3). On the other hand,

$$
\begin{aligned}
t^{1 / 5} f\left(t, x_{t}, y_{t}, z_{t}\right) & =\frac{\Gamma(21 / 20)}{\Gamma(4 / 5)}\left\{3+\int_{-1}^{-t} \frac{4 z^{2}(t+s)+1}{1+z^{2}(t+s)} d s\right\} \\
& =\frac{\Gamma(21 / 20)}{\Gamma(4 / 5)}\left\{3+\int_{t-1}^{0} \frac{4 z^{2}(s)+1}{1+z^{2}(s)} d s\right\} \geq \frac{\Gamma(21 / 20)}{\Gamma(4 / 5)}\left\{3+3 \int_{t-1}^{0} d s\right\} \\
& \geq 3 \frac{\Gamma(21 / 20)}{\Gamma(4 / 5)}=3 \frac{\Gamma(1-\rho+\alpha)}{\Gamma(1-\rho)}, \\
t^{1 / 4} g\left(t, x_{t}, y_{t}, z_{t}\right) & =\frac{\Gamma(5 / 4)}{\Gamma(3 / 4)}\left\{1+\int_{-1}^{-t} \frac{2 x^{2}(t+s)+1}{1+x^{2}(t+s)} d s\right\} \\
& =\frac{\Gamma(5 / 4)}{\Gamma(3 / 4)}\left\{1+\int_{t-1}^{0} \frac{2 x^{2}(s)+1}{1+x^{2}(s)} d s\right\} \geq \frac{\Gamma(5 / 4)}{\Gamma(3 / 4)}\left\{1+\int_{t-1}^{0} d s\right\} \\
& \geq \frac{\Gamma(5 / 4)}{\Gamma(3 / 4)}=\frac{\Gamma(1-\sigma+\beta)}{\Gamma(1-\sigma)}, \quad \\
t^{1 / 2} h\left(t, x_{t}, y_{t}, z_{t}\right) & =\frac{\Gamma(5 / 4)}{\Gamma(1 / 4)}\left\{2+\int_{-1}^{-t} \frac{3 y^{2}(t+s)+1}{1+y^{2}(t+s)} d s\right\} \\
& =\frac{\Gamma(5 / 4)}{\Gamma(1 / 4)}\left\{2+\int_{t-1}^{0} \frac{3 y^{2}(s)+1}{1+y^{2}(s)} d s\right\} \geq \frac{\Gamma(5 / 4)}{\Gamma(1 / 4)}\left\{2+2 \int_{t-1}^{0} d s\right\} \\
& \geq 2 \frac{\Gamma(5 / 4)}{\Gamma(1 / 4)}=2 \frac{\Gamma(1-v+\gamma)}{\Gamma(1-v)} .
\end{aligned}
$$

Hence $\mu=\min \{1,2,3\}=1$ and the above calculations satisfy condition (H4). Then, (3.33) has at least one positive solution.

\section{Unique Existence of Solution}

In this section we give conditions on $f$ and $g$ which render unique positive solution to (1.2).

Theorem 4.1. Let $f, g:(0, T] \times C^{2} \rightarrow[0, \infty)$ be continuous and $\lim _{t \rightarrow 0^{+}} f\left(t, x_{t}, y_{t}\right)=$ $\lim _{t \rightarrow 0^{+}} g\left(t, x_{t}, y_{t}\right)=+\infty$. If there exist $\rho, \sigma \in(0,1)$ such that $0<\rho<\alpha<1,0<\sigma<\beta<1$ and $t^{\rho} f\left(t, x_{t}, y_{t}\right), t^{\sigma} g\left(t, x_{t}, y_{t}\right)$ are continuous functions on $[0, T] \times C^{2}$, then (1.2) has unique positive solution with the following conditions:

(H5) $\left|t^{\rho} f(t, u)-t^{\rho} f(t, v)\right|<L_{1}\|u-v\|,\left|t^{\sigma} g(t, u)-t^{\rho} g(t, v)\right|<L_{2}\|u-v\| ;$

(H6) $L=\max \left\{\left(L_{1} / \Gamma(\alpha)\right) t^{\alpha-\rho} B(1-\rho, \alpha),\left(L_{2} / \Gamma(\beta)\right) t^{\beta-\sigma} B(1-\sigma, \beta)\right\}<1$,

where $u, v \in C^{2}$, and $L_{1}, L_{2}$ are positive constants. 
Proof. As pointed out in the proceeding section, (1.2) is equivalent to the integral equation (3.1) and the solution of (3.1) is equivalent to the fixed point of operator $W$. Thus for $u=$ $\left(u_{1}, v_{1}\right), v=\left(u_{2}, v_{2}\right) \in K$

$$
\begin{aligned}
\left|F u_{1}(t)-F u_{2}(t)\right| & \leq I^{\alpha}\left\{t^{-\rho}\left|t^{\rho} f_{i}\left(t, \bar{u}_{t}+\omega_{t}\right)-t^{\rho} f\left(t, \bar{v}_{t}+\omega_{t}\right)\right|\right\} \\
& \leq L_{1}\|u-v\| I^{\alpha}\left\{t^{-\rho}\right\}=\|u-v\| \frac{L_{1}}{\Gamma(\alpha)} \int_{0}^{t}(t-s)^{\alpha-1} s^{-\rho} \\
& =\frac{L_{1}}{\Gamma(\alpha)}\|u-v\| B(1-\rho, \alpha) t^{\alpha-\rho} .
\end{aligned}
$$

Similarly,

$$
\left|G v_{1}(t)-F v_{2}(t)\right| \leq \frac{L_{2}}{\Gamma(\beta)}\|u-v\| B(1-\sigma, \beta) t^{\beta-\sigma} .
$$

Hence, for each $u, v \in K$, we have

$$
\|W u(t)-W v(t)\|=\max \left\{\max _{0 \leq t \leq T}\left|F u_{1}(t)-F v_{1}(t)\right|,\left|F u_{1}(t)-F v_{1}(t)\right|\right\}=L\|u-v\| .
$$

Hence, by application of the Banach fixed point theorem, $W$ has unique fixed point in $K$, which is the unique positive solution of (1.2).

Example 4.2. Consider the system of nonlinear fractional differential equation

$$
\begin{aligned}
& D^{4 / 5}[x(t)-x(0)]=\frac{1}{\left(1+8 t^{2}\right) \sqrt{t}}\left(x_{t}+y_{t}\right), \quad t \in(0,1], \\
& D^{3 / 4}[y(t)-y(0)]=\frac{1}{\left(1+4 t^{2}\right) \sqrt[4]{t}}\left(x_{t}+y_{t}\right), \quad t \in(0,1], \\
& (x(t), y(t))=\left(-\sin \pi t,-\sin \left(\frac{\pi t}{2}\right)\right), \quad t \in[-1,0],
\end{aligned}
$$

where

$$
x_{t}=\int_{-1}^{-t} \frac{d s}{1+x^{2}(t+s)}, \quad y_{t}=\int_{-1}^{-t} \frac{2 y(t+s)}{1+y^{2}(t+s)} d s
$$


Here, $\alpha=4 / 5, \beta=3 / 4, f\left(t, x_{t}, y_{t}\right)=e^{-t} t^{-1 / 2}\left(x_{t}+y_{t}\right)$, and $g\left(t, x_{t}, y_{t}\right)=e^{-t} t^{-1 / 4}\left(x_{t}+y_{t}\right)$ such that $\lim _{t \rightarrow 0^{+}} f(t, \cdot, \cdot)=\lim _{t \rightarrow 0^{+}} g(t, \cdot, \cdot)=+\infty$. We select $\rho=3 / 4$ and $\sigma=1 / 2$. Hence $t^{\rho} f(t, \cdot, \cdot)$ and $t^{\sigma} g(t, \cdot, \cdot)$ are continuous on $[0,1]$ :

$$
\begin{aligned}
\left|t^{\rho} f\left(t, x_{t}, y_{t}\right)-t^{\sigma} g\left(t, x_{t}, y_{t}\right)\right| \leq & \max \left\{\frac{1}{8 \Gamma(1 / 2)} B\left(\frac{1}{4}, \frac{4}{5}\right), \frac{1}{5 \Gamma(3 / 4)} B\left(\frac{1}{2}, \frac{3}{4}\right)\right\}\|u-v\| \\
& <0.4\|u-v\|,
\end{aligned}
$$

where $u=\left(x_{t}, y_{t}\right), v=\left(x_{t}^{\prime}, y_{t}^{\prime}\right)$. Equation (4.4) satisfies the conditions required in Theorem 4.1 and this theorem implies that the nonlinear equation (4.4) has unique solution in $K$. Using Theorem 3.2, (4.4) is equivalent to the system of integral equations

$$
\begin{gathered}
x(t)=x(0)+I^{4 / 5}\left\{\frac{1}{\left(1+8 t^{2}\right) \sqrt{t}}\left(x_{t}+y_{t}\right)\right\}, \quad t \in(0,1], \\
y(t)=y(0)+I^{3 / 4}\left\{\frac{1}{\left(1+4 t^{2}\right) \sqrt[4]{t}}\left(x_{t}+y_{t}\right)\right\}, \quad t \in(0,1], \\
(x(t), y(t))=\left(-\sin \pi t,-\sin \frac{\pi}{2} t\right), \quad t \in[-1,0],
\end{gathered}
$$

and the solution of $(4.4)$ is $(x(t), y(t))=\lim _{n \rightarrow+\infty}\left(x^{n}(t), y^{n}(t)\right)$ where

$$
\underbrace{\left(x^{n+1}(t), y^{n+1}(t)\right)}_{n=0,1,2, \ldots}=\left(F x^{n}(t), G y^{n}(t)\right), \quad x^{0}(t)=x(0), \quad y^{0}(0)=y(0)
$$

\section{Conclusions}

In this paper the existence of positive solutions for system of nonlinear fractional differential equations in two dimensions with the delay comprising of standard Riemann-Liouville fractional derivatives has been discussed in Banach space. The unique solution under Lipschitz condition is also derived. For getting our results in this paper Leray-Schauder's theorem, Krasnoselskii's fixed point theorem, and Banach contraction principle had played important roles. Our research work in this paper can be generalized to system of nonlinear fractional differential equations in multiple dimensions with the finite delay or infinite delay. The present work provides insights in the equations encountered in fractional order dynamic systems and the control systems which further may be explored.

\section{Acknowledgments}

The authors thank the referees' efforts for their remarks and Professor Dumitru Băleanu for his regular contact. 


\section{References}

[1] I. Podlubny, Fractional Differential Equations: An Introduction to Fractional Derivatives, Fractional Differential Equations, to Methods of Their Solution and Some of Their Application, vol. 198 of Mathematics in Science and Engineering, Academic Press, San Diego, Calif, USA, 1999.

[2] K. B. Miller and B. Ross, An Introduction to the Fractional Calculus and Fractional Differential Equations, Wiely, New York, NY, USA, 1993.

[3] K. B. Oldham and I. Spanier, The Fractional Calculus, Academic Press, Londan, UK, 1997.

[4] S. G. Samko, A. A. Kilbas, and O. I. Marichev, Fractional Integrals and Derivatives: Theory and Applications, Gordon and Breach Science, Yverdon, Switzerland, 1993.

[5] B. J. West, M. Bologna, and P. Grigolini, Eds., Physics of Fractal Operators, Springer, New York, NY, USA, 2003.

[6] J. K. Hale and S. M. Verduyn Lunel, Introduction to Functional-Differential Equations, vol. 99 of Applied Mathematical Sciences, Springer, New York, NY, USA, 1993.

[7] J. Ander and P. Seibert, "Ü ber die stückweise linearen differentiallgleichungen, die bei regelungsproblemen auftreten," Archiv der Mathematik, vol. 7, no. 3, pp. 148-164, 1956.

[8] S. V. Drakunov and V. I. Utkin, "Sliding mode control in dynamic systems," International Journal of Control, vol. 55, no. 4, pp. 1029-1037, 1992.

[9] E. Shustin, "Dynamics of oscillations in a multi-dimensional delay differential system," Discrete and Continuous Dynamical Systems. Series A, vol. 11, no. 2-3, pp. 557-576, 2004.

[10] S. Roy, A. Saberi, and Y. Wan, "On multiple-delay output feedback stabilization of LTI plants," International Journal of Robust and Nonlinear Control. In press.

[11] A. Oustaloup, Systèms Asservis Lineaires d'Ordre Fractionnaire, Masson, Paris, France, 1983.

[12] A. Oustaloup, La Command CRONE, série Automatique, Hermès, Paris, France, 1991.

[13] V. Daftardar-Gejji and A. Babakhani, "Analysis of a system of fractional differential equations," Journal of Mathematical Analysis and Applications, vol. 293, no. 2, pp. 511-522, 2004.

[14] V. Daftardar-Gejji and H. Jafari, "Analysis of a system of nonautonomous fractional differential equations involving Caputo derivatives," Journal of Mathematical Analysis and Applications, vol. 328, no. 2, pp. 1026-1033, 2007.

[15] M. C. Joshi and R. K. Bose, Some Topics in Nonlinear Functional Analysis, A Halsted Press Book, Wiley Eastern Limited, New Dehli, India, 1985.

[16] M. A. Krasnoselskiř, Positive Solutions of Operator Equations, P. Noordhoff, Groningen, The Netherlands, 1964.

[17] A. Babakhani and V. Daftardar-Gejji, "Existence of positive solutions of nonlinear fractional differential equations," Journal of Mathematical Analysis and Applications, vol. 278, no. 2, pp. 434-442, 2003.

[18] C.-Z. Bai and J.-X. Fang, "The existence of a positive solution for a singular coupled system of nonlinear fractional differential equations," Applied Mathematics and Computation, vol. 150, no. 3, pp. 611-621, 2004. 


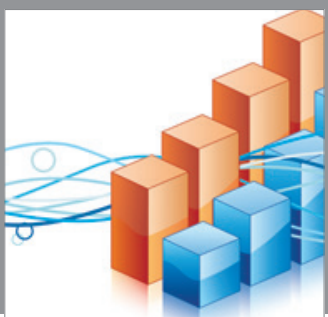

Advances in

Operations Research

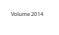

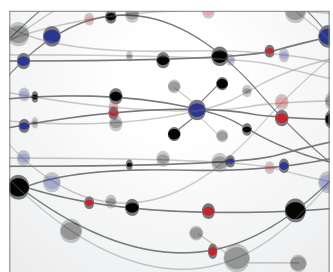

\section{The Scientific} World Journal
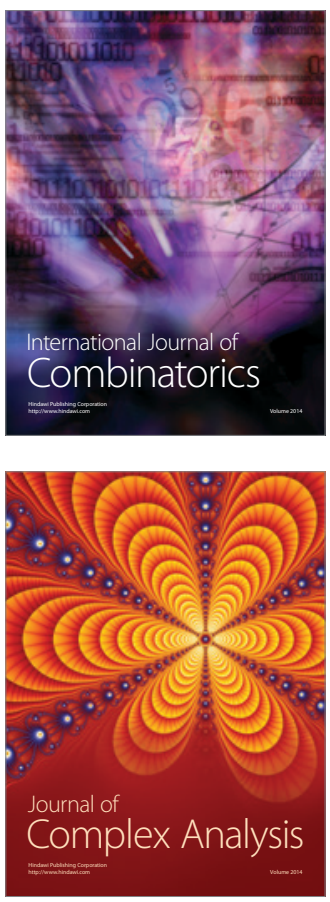

International Journal of

Mathematics and

Mathematical

Sciences
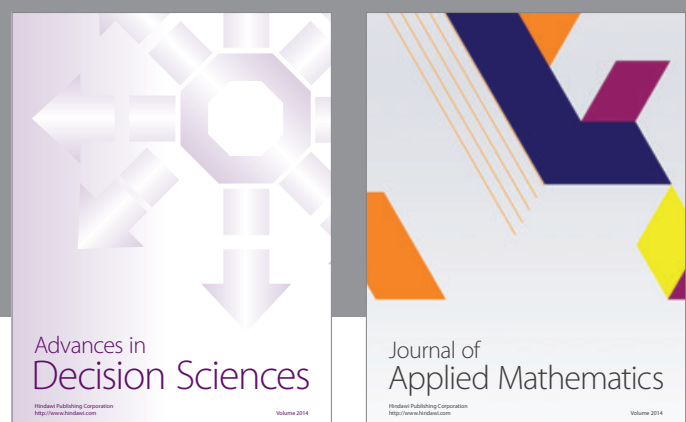

Journal of

Applied Mathematics
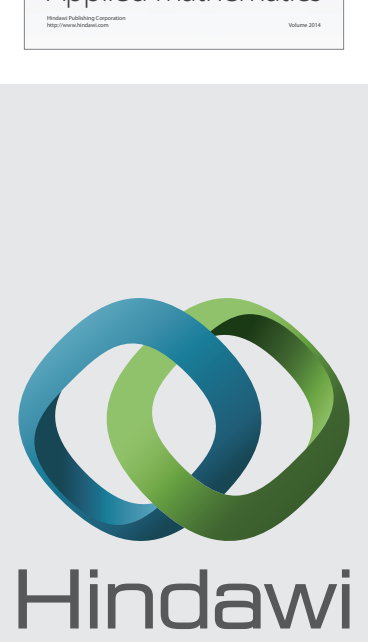

Submit your manuscripts at http://www.hindawi.com
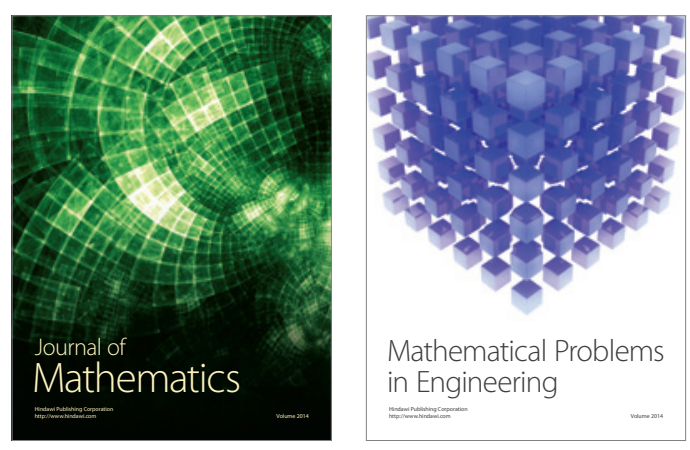

Mathematical Problems in Engineering
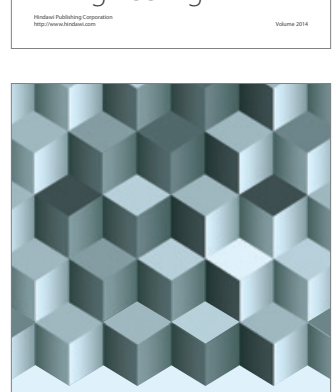

Journal of

Function Spaces
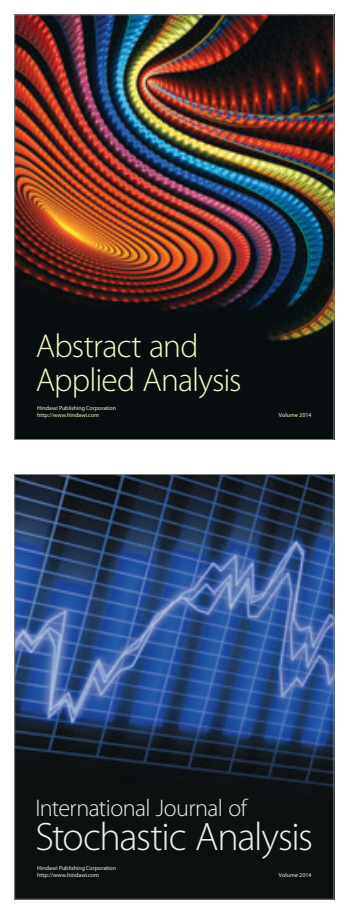

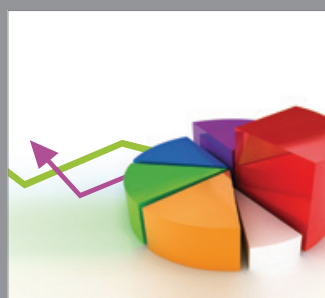

ournal of

Probability and Statistics

Promensencen
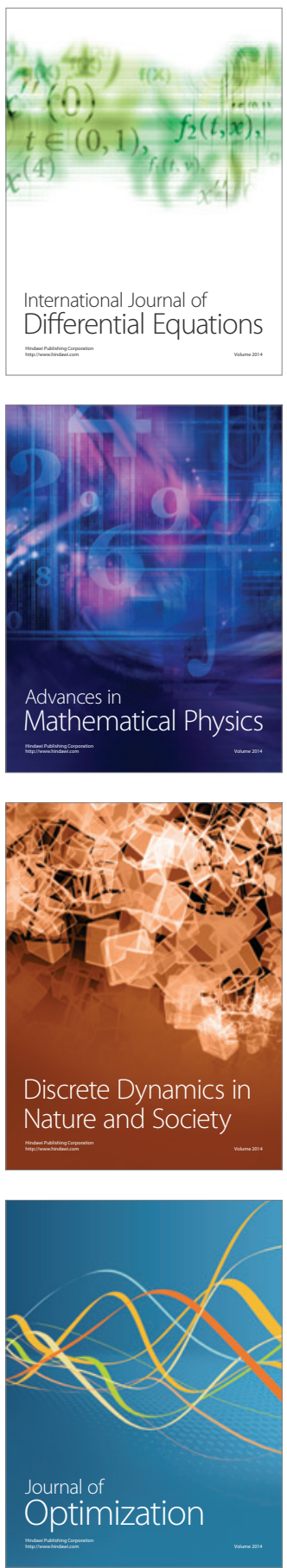